TRANSACTIONS OF THE

AMERICAN MATHEMATICAL SOCIETY

Volume 348, Number 9, September 1996

\title{
ON REPRESENTATIONS OF AFFINE KAC-MOODY GROUPS AND RELATED LOOP GROUPS
}

\author{
YU CHEN
}

Dedicated to Professor G. Zacher on the occasion of his seventieth birthday

\begin{abstract}
We demonstrate a one to one correspondence between the irreducible projective representations of an affine Kac-Moody group and those of the related loop group, which leads to the results that every non-trivial representation of an affine Kac-Moody group must have its degree greater than or equal to the rank of the group and that the equivalence appears if and only if the group is of type $A_{n}^{(1)}$ for some $n \geq 1$. Moreover the characteristics of the base fields for the non-trivial representations are found being always zero.
\end{abstract}

\section{INTRODUCTION AND MAIN THEOREM}

Let $\tilde{\mathfrak{g}}$ be a non-twisted affine Kac-Moody algebra over a field $F$ of characteristic zero and let $\Phi$ be its real root system with respect to a fixed Cartan subalgebra. Denote by $\tilde{\mathfrak{g}}_{a}$ the root subspace of $\tilde{\mathfrak{g}}$ related to a root $a \in \Phi$. Let $G^{*}$ be the free product of the additive groups $\tilde{\mathfrak{g}}_{a}$ for all $a \in \Phi$ and $\iota: \tilde{\mathfrak{g}}_{a} \rightarrow G^{*}$ the canonical embedding. Set $\tilde{\mathfrak{g}}^{\prime}=[\tilde{\mathfrak{g}}, \tilde{\mathfrak{g}}]$. For any integrable $\tilde{\mathfrak{g}}^{\prime}$-module $V$, or $(V, \psi)$ with $\psi: \tilde{\mathfrak{g}}^{\prime} \rightarrow$ $\operatorname{End}_{F}(V)$, let $\psi^{*}: G^{*} \rightarrow G L(V)$ be the homomorphism defined by

$$
\psi^{*}(\iota(e))=\exp \psi(e), \quad a \in \Phi, e \in \tilde{\mathfrak{g}}_{a},
$$

where exp is the canonical exponential map. Suppose $N$ is the intersection of all ker $\psi^{*}$. The quotient group of $G^{*}$ modulo $N$, denoted by $\tilde{G}(F)$, is called an affine (non-twisted) Kac-Moody group and the real root system $\Phi$ is also called a root system of $\tilde{G}(F)$. This approach of defining $\tilde{G}(F)$ is due to Kac and Peterson (see [8] and [10]). A review of other approaches can be found in Tits' survey paper [13]. The main aim of this paper is to show that the minimal representation degree of an affine Kac-Moody group over a field of characteristic zero depends only on the number of the fundamental roots of the group. This number is called the rank of the group and is denoted by, if the group is $\tilde{G}(F), \operatorname{rank} \tilde{G}$. In the following, for a representation we always mean a linear or projective representation of finite degree over a field, unless otherwise indicated. Our main result is as follows.

Theorem. Let $\tilde{G}(F)$ be an affine Kac-Moody group. Then

Received by the editors August 4, 1995.

1991 Mathematics Subject Classification. Primary 17B67, 20C15, 22E70.

Key words and phrases. Kac-Moody group, loop group, Chevalley-Demazure group scheme, minimal representation.

Research supported in part by the Italian M.U.R.S.T. and C.N.R.-G.N.S.A.G.A.

(C)1996 American Mathematical Society 
(i) the degree of every non-trivial representation of $\tilde{G}(F)$ must be greater than or equal to $\operatorname{rank} \tilde{G}$;

(ii) the base fields of non-trivial representations of $\tilde{G}(F)$ are always of characteristic zero;

(iii) there exists a non-trivial representation of $\tilde{G}(F)$ with degree equal to rank $\tilde{G}$ if and only if the associated Kac-Moody algebra $\tilde{\mathfrak{g}}$ is of the non-twisted type $A_{n}^{(1)}$ (see [7] for the notation) for $n \geq 1$.

It is known that affine Kac-Moody groups are very closely related with loop groups and this close relation extends to their representations, as exposed for instance in [11] for the case when $F$ is the real number field or the complex number field. For an arbitrary field $F$ of characteristic zero, we establish an explicit one-toone correspondence between the irreducible projective representations of an affine Kac-Moody group and the irreducible projective representations of the related loop group, which enable us to deduce our result from exploring the representations of the related loop groups. Apart from their applications to affine Kac-Moody groups, the properties of these loop groups certainly have their own interests. In fact, the representations of these loop groups are found always having degrees strictly greater than their ranks (see Proposition 3.4). Moreover, such a loop group, whenever simply connected, has a nontrivial representation whose degree is equal to its rank plus one if and only if it is isomorphic to the special linear group $S L_{n}\left(F\left[t, t^{-1}\right]\right)$, where $F\left[t, t^{-1}\right]$ is the Laurent polynomial ring over $F$ and $n>1$ (see Theorem 3.5).

\section{Projective Representations of KaC-Moody groups AND RELATED LOOP GROUPS}

Throughout this paper, we fix a field $F$ of characteristic zero and the affine Kac-Moody algebra $\tilde{\mathfrak{g}}$ is always over $F$. The additive group and the multiplicative group of $F$ are denoted by $F^{+}$and $F^{*}$ respectively. Let $\tilde{A}$ be the generalized Cartan matrix associated to $\tilde{\mathfrak{g}}$, that is $\tilde{A}=\left(A_{i j}\right)$, where $A_{i j} \in \mathbb{Z}$ for $i, j \in\{0,1, \ldots, \ell\}$ such that

$$
A_{i i}=2 ; A_{i j} \leq 0 \text { if } i \neq j ; \quad A_{i j}=0 \Longleftrightarrow A_{j i}=0 .
$$

For convenience we assume that $\tilde{A}$ is indecomposible. Suppose $\Delta=\left\{a_{0}, a_{1}, \ldots, a_{\ell}\right\}$ is a fundamental root system of $\Phi$. We denote by $W$ the Weyl group of $\Phi$, which is generated by the fundamental reflections $\left\{s_{0}, s_{1}, \ldots, s_{\ell}\right\}$ satisfying

$$
s_{i}\left(a_{j}\right)=a_{j}-A_{i j} a_{i}, \quad 0 \leq i, j \leq \ell .
$$

A pair of real roots $a, b \in \Phi$ is called prenilpotent if there exist elements $r, r^{\prime} \in W$ such that

$$
r(a), r(b) \in\left\{\sum_{i=0}^{\ell} n_{i} a_{i} \mid n_{i} \in \mathbb{N}\right\} ; r^{\prime}(a), r^{\prime}(b) \in\left\{-\sum_{i=0}^{\ell} n_{i} a_{i} \mid n_{i} \in \mathbb{N}\right\} .
$$

Let $a d: \tilde{\mathfrak{g}} \rightarrow \operatorname{End}_{F}(\tilde{\mathfrak{g}})$ be the adjoint representation of $\tilde{\mathfrak{g}}$. For each $a \in \Phi$, it is possible to choose a base vector $\tilde{e}_{a}$ in the root subspace $\tilde{\mathfrak{g}}_{a}$ such that (see [6], §6)

$$
\exp a d\left(\tilde{e}_{a_{i}}\right) \cdot \exp a d\left(-\tilde{e}_{-a_{i}}\right) \cdot \exp a d\left(\tilde{e}_{a_{i}}\right)\left(e_{a}\right)=\tilde{e}_{s_{i}(a)} \text { or }-\tilde{e}_{s_{i}(a)}, \quad 0 \leq i \leq \ell .
$$

We define the number $n_{i, a}$ by

$$
\exp \operatorname{ad}\left(\tilde{e}_{a_{i}}\right) \cdot \exp \operatorname{ad}\left(-\tilde{e}_{-a_{i}}\right) \cdot \exp \operatorname{ad}\left(\tilde{e}_{a_{i}}\right)\left(e_{a}\right)=n_{i, a} \tilde{e}_{s_{i}(a)} .
$$


Let $\gamma: G^{*} \rightarrow \tilde{G}(F)$ be the natural homomorphism. For each $a \in \Phi$, we denote by $\lambda_{a}: F^{+} \rightarrow \tilde{\mathfrak{g}}_{a}$ the homomorphism satisfying $\lambda_{a}(q)=q \tilde{e}_{a}$ for all $q \in F^{+}$and write $\tilde{u}_{a}$ for the composite $\iota_{a} \gamma \lambda_{a}$. We define, for each $q \in F^{*}$ and $0 \leq i \leq \ell$,

$$
\tilde{w}_{i}(q)=\tilde{u}_{a_{i}}(q) \tilde{u}_{-a_{i}}\left(-q^{-1}\right) \tilde{u}_{a_{i}}(q) ; \quad \tilde{h}_{i}(q)=\tilde{w}_{i}(q) \tilde{w}_{i}(1)^{-1} .
$$

Proposition 2.1. The Kac-Moody group $\tilde{G}(F)$ is generated by the elements $u_{a}(q)$ for all $a \in \Phi$ and $q \in F$ subject to the following relations:

(i) $\tilde{h}_{i}(p) \tilde{h}_{i}(q)=\tilde{h}_{i}(p q)$ for $p, q \in F^{*}$;

(ii) $\tilde{h}_{i}(p) \tilde{h}_{j}(q)=\tilde{h}_{j}(q) \tilde{h}_{i}(p)$ for $i, j \in\{0,1, \ldots, \ell\}, p, q \in F^{*}$;

(iii) $\tilde{u}_{a}(p) \tilde{u}_{a}(q)=\tilde{u}_{a}(p+q)$ for $a \in \Phi, p, q \in F$

(iv) $\tilde{h}_{j}(q) \tilde{u}_{a_{i}}(p) \tilde{h}_{j}(q)^{-1}=\tilde{u}_{a_{i}}\left(q^{A_{j i}} p\right)$ for $p \in F$ and $q \in F^{*}$;

(v) $\tilde{w}_{i}(1) \tilde{h}_{j}(q) \tilde{w}_{i}(1)^{-1}=\tilde{h}_{j}(q) \tilde{h}_{i}\left(q^{-A_{j i}}\right)$ for $q \in F^{*}$;

(vi) $\tilde{w}_{i}(1) \tilde{u}_{a}(p) \tilde{w}_{i}(1)^{-1}=u_{s_{i}(a)}\left(n_{i, a} p\right)$ for $a \in \Phi$ and $p \in F$;

(vii) for every prenilpotent pair $a, b \in \Phi$,

$$
\left[\tilde{u}_{a}(p), \tilde{u}_{b}(q)\right]=\prod_{\substack{i, j \in \mathbb{N}-\{0\} \\ i a+j b \in \Phi}} \tilde{u}_{i a+j b}\left(C_{i j a b} p^{i} q^{j}\right), \quad p, q \in F,
$$

where $C_{i j a b}$ is an integer determined uniquely by $i, j, a, b$ and the order in which the terms on the right are taken.

Proof. It is obvious that $\left\{\tilde{u}_{a}(q) \mid q \in F, a \in \Phi\right\}$ is a set of generators of $\tilde{G}(F)$ by the definition. The above relations come from the relations given by Tits in [14] (see also [2]).

Since $\tilde{\mathfrak{g}}$ is of non-twisted type, we may assume that $\tilde{A}=\left(A_{i j}\right)(i, j \in\{0,1, \ldots, \ell\})$ is the extended matrix of a Cartan matrix $A=\left(A_{i j}\right)(i, j \in\{1,2, \ldots, \ell\})$. Suppose $\mathfrak{g}_{\mathbb{C}}$ is a simple complex Lie algebra whose Cartan matrix is $A$. Then the subset $\Delta_{0}=\left\{a_{1}, a_{2}, \ldots, a_{\ell}\right\} \subset \Delta$ is a fundamental root system of $\mathfrak{g}_{\mathbb{C}}$ while

$$
\Phi_{0}=\Phi \cap\left\{\sum_{i=1}^{\ell} n_{i} a_{i} \mid n_{i} \in \mathbb{Z}\right\}
$$

is a root system of $\mathfrak{g}_{\mathbb{C}}$. In particular, the Weyl group $W_{0}$ of $\Phi_{0}$ is generated by the reflections $\left\{s_{1}, s_{2}, \ldots, s_{\ell}\right\} \subset W$. Let $d$ be the highest root of $\Phi_{0}$ and let $\delta=a_{0}+d$. Then we have

$$
\Phi=\left\{a+m \delta \mid a \in \Phi_{0}, m \in \mathbb{Z}\right\}
$$

(see [7], Ch.6). Suppose $\mathfrak{g}$ is a $\mathbb{Z}$-form of $\mathfrak{g}_{\mathbb{C}}$ with Chevalley generators $\left\{e_{a} \mid a \in \Phi_{0}\right\}$. Then there is a central extension (cf. [7])

$$
0 \longrightarrow F \longrightarrow \tilde{\mathfrak{g}}^{\prime} \stackrel{\theta}{\longrightarrow} \mathfrak{g} \otimes_{\mathbb{Z}} F\left[t, t^{-1}\right] \longrightarrow 0
$$

such that

$$
\theta\left(\tilde{e}_{a+m \delta}\right)=e_{a} \otimes t^{m}, \quad a \in \Phi_{0}, m \in \mathbb{Z} .
$$

Moreover, let $\mathfrak{c}$ be the centre of $\tilde{\mathfrak{g}}^{\prime}$, then $\theta$ induces canonically an isomorphism

$$
\theta^{*}: \operatorname{Aut}_{F}\left(\tilde{\mathfrak{g}}^{\prime} / \mathfrak{c}\right) \rightarrow \operatorname{Aut}_{F}\left(\mathfrak{g} \otimes_{\mathbb{Z}} F\left[t, t^{-1}\right]\right) .
$$

Let $A d: \tilde{G}(F) \rightarrow A u t_{F}(\tilde{\mathfrak{g}})$ be the adjoint representation of $\tilde{G}(F)$. Since $\tilde{G}(F)$ acts trivially on $\mathfrak{c}, A d$ induces a homomorphism from $\tilde{G}(F)$ to $A u t_{F}(\tilde{\mathfrak{g}} / \mathfrak{c})$ through the natural homomorphism $\tilde{\mathfrak{g}} \rightarrow \tilde{\mathfrak{g}} / \mathfrak{c}$, which is denoted also by $A d$ without confusion. 
Lemma 2.2. Let $G_{a d}$ be an adjoint Chevalley-Demazure group scheme associated to $\mathfrak{g}_{\mathbb{C}}$. Then there exists a homomorphism $\alpha$ from $\tilde{G}(F)$ to $G_{a d}\left(F\left[t, t^{-1}\right]\right)$ such that the following diagram is commutative

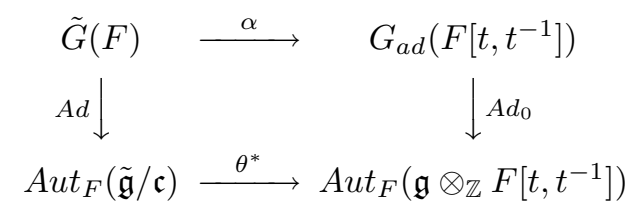

where $A d_{0}$ is the adjoint representation of $G_{a d}\left(F\left[t, t^{-1}\right]\right)$.

Proof. Note that for each $a \in \Phi_{0}$, there is a canonical homomorphism $\bar{u}_{a}$ from the additive group $F\left[t, t^{-1}\right]^{+}$of $F\left[t, t^{-1}\right]$ to $G_{a d}\left(F\left[t, t^{-1}\right]\right)$ satisfying

$$
\begin{aligned}
& \operatorname{Ad}_{0} \bar{u}_{a}(x)(e)=\operatorname{expad}_{0}\left(x e_{a}\right) \cdot e \cdot\left(\operatorname{expad}_{0}\left(x e_{a}\right)\right)^{-1}, \\
& x \in F\left[t, t^{-1}\right], e \in \mathfrak{g} \otimes_{\mathbb{Z}} F\left[t, t^{-1}\right],
\end{aligned}
$$

where $a d_{0}$ is the adjoint representation of $\mathfrak{g} \otimes_{\mathbb{Z}} F\left[t, t^{-1}\right]$ (cf. [5] and [9], §2). Since $\tilde{G}(F)$ is generated by the elements $\tilde{u}_{a}(q)$ for all $a \in \Phi_{0}$ and $q \in F$, we define the map $\alpha$ by

$$
\alpha\left(\tilde{u}_{a+m \delta}(q)\right)=\bar{u}_{a}\left(q t^{m}\right), \quad a \in \Phi_{0}, m \in \mathbb{Z} .
$$

By using Proposition 2.1, one can easily check that $\alpha$ is a homomorphism and the above diagram is commutative.

Let $G$ be a simply connected Chevalley-Demazure group scheme associated to $\mathfrak{g}_{\mathbb{C}}$. Then $G\left(F\left[t, t^{-1}\right]\right)$ is a loop group. Denote again by $A d_{0}$ the adjoint representation of $G\left(F\left[t, t^{-1}\right]\right)$ and by $u_{a}: F\left[t, t^{-1}\right]^{+} \rightarrow G\left(F\left[t, t^{-1}\right]\right)$ the canonical exponential homomorphism which satisfies

$$
\begin{aligned}
& \operatorname{Ad}_{0} u_{a}(x)(e)=\operatorname{expad}_{0}\left(x e_{a}\right) \cdot e \cdot\left(\operatorname{expad}_{0}\left(x e_{a}\right)\right)^{-1}, \\
& \qquad x \in F\left[t, t^{-1}\right], e \in \mathfrak{g} \otimes_{\mathbb{Z}} F\left[t, t^{-1}\right] .
\end{aligned}
$$

For each root $a \in \Phi_{0}$, let $a^{\vee}$ be the coroot of $a$. Denote by $\Phi_{0}^{\vee}$ the set of coroots and by $\langle\rangle:, \Phi_{0} \times \Phi_{0}^{\vee} \rightarrow \mathbb{Z}$ the canonical bilinear form which satisfies

$$
\left\langle a_{i}, a_{j}^{\vee}\right\rangle=A_{j i} \text { for } i, j \in\{1,2, \ldots, \ell\} .
$$

Lemma 2.3. Suppose $x \in F\left[t, t^{-1}\right]^{*}$. Let $w_{i}(x)=u_{a_{i}}(x) u_{-a_{i}}\left(-x^{-1}\right) u_{a_{i}}(x)$ for $1 \leq i \leq \ell, w_{0}(x)=u_{-d}(x) u_{d}\left(-x^{-1} x\right) u_{-d}(x)$ and $h_{i}(x)=w_{i}(x) w_{i}(1)$ for $0 \leq i \leq \ell$. Then

(i) $h_{0}(x) h_{0}(y)=h_{0}(x y)$ for $y \in F\left[t, t^{-1}\right]^{*}$;

(ii) $h_{0}(x) h_{i}(y)=h_{i}(y) h_{0}(x)$ for $i \in\{1,2, \ldots, \ell\}, y \in F\left[t, t^{-1}\right]^{*}$;

(iii) for $y \in F\left[t, t^{-1}\right]$, we have

$$
h_{0}(x) u_{a_{i}}(y) h_{0}(x)^{-1}=u_{a_{i}}\left(q^{A_{0 i}} y\right), \quad 1 \leq i \leq \ell,
$$

and

$$
h_{j}(x) u_{-d}(y) h_{j}(x)^{-1}=u_{-d}\left(q^{A_{j 0}} y\right), \quad 0 \leq j \leq \ell ;
$$

(iv) $w_{i}(1) h_{j}(x) w_{i}(1)^{-1}=h_{j}(x) h_{i}\left(x^{-A_{j i}}\right)$ for $0 \leq i \leq \ell$ and $0 \leq j \leq \ell$; 
(v) $w_{i}(1) u_{a}(y) w_{i}(1)^{-1}=u_{s_{i}(a)}\left(n_{i, a} y\right)$, for $a \in \Phi_{0}, y \in F\left[t, t^{-1}\right]$ and $1 \leq i \leq \ell$ while

$$
w_{0}(1) u_{a}(y) w_{0}(1)^{-1}=u_{a-\left\langle a, d^{\vee}\right\rangle d}\left(n_{0, a} y\right),
$$

where $n_{i, a}= \pm 1$ is the same number as in Proposition 2.1 (vi) and, moreover,

$$
n_{i, a}=n_{i, a+m \delta}, \quad 0 \leq i \leq \ell, m \in \mathbb{Z}
$$

(vi) for each prenilpotent pair $a, b \in \Phi_{0}$ and $y, z \in F\left[t, t^{-1}\right]$,

$$
\left[u_{a}(y), u_{b}(z)\right]=\prod_{\substack{i, j \in \mathbb{N}-\{0\} \\ i a+j b \in \Phi_{0}}} u_{i a+j b}\left(C_{i j a b} y^{i} z^{j}\right),
$$

where $C_{i j a b}$ and the order in which the terms on the right are taken are the same as in (2.1). Moreover, for $m, n \in \mathbb{Z}$,

$$
C_{i j a b}=C_{i j, a+m \delta, b+n \delta} .
$$

Proof. Suppose the coroot $d^{\vee}$ of the highest root $d$ has an expression

$$
d^{\vee}=m_{1} a_{1}^{\vee}+m_{2} a_{2}^{\vee}+\cdots+m_{\ell} a_{\ell}^{\vee},
$$

where $m_{i} \in \mathbb{N}$ for $1 \leq i \leq \ell$. Then we have

$$
h_{0}(x)=\prod_{i=1}^{\ell} h_{a_{i}}\left(x^{-m_{i}}\right),
$$

from which follows (i) and (ii).

Let

$$
d=n_{1} a_{1}+n_{2} a_{2}+\cdots+n_{\ell} a_{\ell}
$$

be the expression of $d$ in terms of the fundamental roots. Since $\tilde{A}=\left(A_{i j}\right)(i, j \in$ $\{0,1, \ldots, \ell\})$ is the extended Cartan matrix of $\left(A_{i j}\right)(i, j \in\{1,2, \ldots, \ell\})$, we have for all $0 \leq i \leq \ell$

$$
-A_{0 i}=m_{1} A_{1 i}+m_{2} A_{2 i}+\cdots+m_{\ell} A_{\ell i}
$$

and

$$
-A_{i 0}=n_{1} A_{i 1}+n_{2} A_{i 2}+\cdots+n_{\ell} A_{i \ell} .
$$

Hence the expressions (2.7) and (2.8) yield for $1 \leq i \leq \ell$

$$
\begin{aligned}
h_{0}(x) u_{a_{i}}(y) h_{0}(x)^{-1} & =u_{a_{i}}\left(\prod_{j=1}^{\ell}\left(x^{-m_{j}}\right)^{\left\langle a_{i}, a_{j}^{\vee}\right\rangle} y\right) \\
& =u_{a_{i}}\left(x^{-\sum_{j=1}^{\ell} m_{j} A_{j i}} y\right)=u_{a_{i}}\left(x^{A_{0 i}} y\right),
\end{aligned}
$$

while (2.8) and (2.9) yield

$$
\begin{aligned}
h_{i}(x) u_{d}(y) h_{i}(x)^{-1} & =u_{d}\left(x^{-\left\langle d, a_{i}^{\vee}\right\rangle} y\right)=u_{d}\left(x^{-\sum_{j=1}^{\ell} n_{j}\left\langle a_{j}, a_{i}^{\vee}\right\rangle} y\right) \\
& =u_{d}\left(x^{-\sum_{j=1}^{\ell} n_{j} A_{i j}} y\right)=u_{d}\left(x^{A_{i 0}} y\right),
\end{aligned}
$$

where $x \in F\left[t, t^{-1}\right]^{*}, y \in F\left[t, t^{-1}\right]$. Moreover, we have by (2.8) and (2.10)

$$
h_{0}(x) u_{d}(y) h_{0}(x)^{-1}=u_{d}\left(x^{-\sum_{j=1}^{\ell} m_{j} A_{j 0}} y\right)=u_{d}\left(x^{A_{00}} y\right) .
$$

Thus (iii) is proved. 
For showing (iv), it is sufficient to show the following two identities:

$$
w_{0}(1) h_{i}(x) w_{0}(1)^{-1}=h_{i}(x) h_{0}\left(x^{-A_{i 0}}\right), \quad 1 \leq i \leq \ell,
$$

and

$$
w_{i}(1) h_{0}(x) w_{i}(1)^{-1}=h_{0}(x) h_{i}\left(x^{-A_{0 i}}\right), \quad 0 \leq i \leq \ell .
$$

In fact, it follows from (iii) that

$$
\begin{aligned}
h_{i}(x)^{-1} w_{0}(1) h_{i}(x) & =h_{i}(x)^{-1} u_{-d}(1) u_{d}(-1) u_{-d}(1) h_{i}(x) \\
& =u_{-d}\left(x^{A_{i 0}}\right) u_{d}\left(-x^{A_{i 0}}\right) u_{-d}\left(x^{-A i 0}\right) \\
& =w_{0}\left(x^{-A_{i 0}}\right)=h_{0}\left(x^{-A i 0}\right) w_{0}(1),
\end{aligned}
$$

which implies the identity (2.12) immediately. For $0 \leq i \leq \ell$, we have by $(2.8)$

$$
\begin{aligned}
w_{i}(1) h_{0}(x) w_{i}(1)^{-1} & =w_{i}(1)\left(\prod_{j=1}^{\ell} h_{j}\left(x^{-m_{j}}\right)\right) w_{i}(1)^{-1}=\prod_{j=1}^{\ell} h_{j}\left(x^{-m_{j}}\right) h_{i}\left(x^{-m_{j} A_{i j}}\right) \\
& =h_{0}(x) h_{i}\left(x^{-\sum_{j=1}^{\ell} m_{j} A_{i j}}\right)=h_{0}(x) h_{i}\left(x^{-A_{i 0}}\right),
\end{aligned}
$$

from which follows (2.13).

Now we come to show (v). For each element $g \in G\left(F\left[t, t^{-1}\right]\right)$, we denote by $\bar{g}$ the image of $g$ under the natural homomorphism $G\left(F\left[t, t^{-1}\right]\right) \rightarrow G_{a d}\left(F\left[t, t^{-1}\right]\right)$. Given a root $a \in \Phi_{0}$, we have

$$
\bar{w}_{i}(1) \bar{u}_{a}(x) \bar{w}_{i}(1)^{-1}=\bar{u}_{s_{i}(a)}\left(n_{i, a}^{\prime} x\right), \quad x \in F\left[t, t^{-1}\right], 1 \leq i \leq \ell,
$$

where $n_{i, a}^{\prime}$ is an integer determined by $i$ and $a$. On the other hand, we have by Proposition 2.1 (vi) and Lemma 2.2 that for all $q \in F$ and $1 \leq i \leq \ell$

$$
\begin{aligned}
\bar{w}_{i}(1) \bar{u}_{a}(q) \bar{w}_{i}(1)^{-1} & =\alpha\left(\tilde{w}_{i}(1) \tilde{u}_{a}(q) \tilde{w}_{i}(1)^{-1}\right) \\
& =\alpha\left(\tilde{u}_{s_{i}(a)}(q)\right)=\bar{u}_{s_{i}(a)}\left(n_{i, a} q\right) .
\end{aligned}
$$

Comparing this identity with (2.14), we obtain that $n_{i, a}=n_{i, a}^{\prime}$ for all $a \in \Phi_{0}$ and $1 \leq i \leq \ell$. Moreover, we have for $1 \leq i \leq \ell, m \in \mathbb{Z}, a \in \Phi_{0}$ and $q \in F$

$$
\begin{aligned}
\bar{u}_{s_{i}(a)}\left(n_{i, a} q t^{m}\right) & =\bar{w}_{i}(1) \bar{u}_{a}\left(q t^{m}\right) \bar{w}_{i}(1)^{-1}=\alpha\left(\tilde{w}_{i}(1) \tilde{u}_{a+m \delta}(q) \tilde{w}_{i}(1)^{-1}\right) \\
& =\alpha\left(\tilde{u}_{s_{i}(a+m \delta)}\left(n_{i, a+m \delta} q\right)\right)=\bar{u}_{s_{i}(a)}\left(n_{i, a+m \delta} q t^{m}\right),
\end{aligned}
$$

from which follows the identity (2.4) for $1 \leq i \leq \ell$. Note that for $a \in \Phi_{0}$, we have

$$
\left\langle\delta, a_{0}^{\vee}\right\rangle=0
$$

and

$$
\left\langle a, d^{\vee}\right\rangle=-\left\langle a, a_{0}^{\vee}\right\rangle
$$

Hence

$$
s_{0}(a+m \delta)=a+m \delta-\left\langle a, a_{0}^{\vee}\right\rangle a_{0}=a-\left\langle a, d^{\vee}\right\rangle d+\left(m+\left\langle a, d^{\vee}\right\rangle\right) \delta .
$$

Thus we obtain from Proposition 2.1 (vi) and Lemma 2.2 that

$$
\begin{aligned}
\bar{w}_{0}(t) \bar{u}_{a}\left(q t^{m}\right) \bar{w}_{0}(t)^{-1} & =\alpha\left(\tilde{w}_{0}(1) \tilde{u}_{a+m \delta}(q) \tilde{w}_{0}(1)^{-1}\right) \\
& =\alpha\left(\tilde{u}_{s_{0}(a+m \delta)}\left(n_{0, a+m \delta} q\right)\right) \\
& =\bar{u}_{a-\left\langle a, d^{\vee}\right\rangle d}\left(n_{0, a+m \delta} q t^{m+\left\langle a, d^{\vee}\right\rangle}\right) .
\end{aligned}
$$


However, it is known that for all $a \in \Phi_{0}$ and $x \in F\left[t, t^{-1}\right]$

$$
\bar{w}_{0}(t) \bar{u}_{a}(x) \bar{w}_{0}(t)^{-1}=\bar{u}_{a-\left\langle a, d^{\vee}\right\rangle d}\left(n^{\prime} x t^{\left\langle a, d^{\vee}\right\rangle}\right),
$$

where $n^{\prime} \in\{ \pm 1\}$ is determined by the roots $a$ and $d$ (cf. [9]). Comparing this identity with (2.15), we obtain that for all $m \in \mathbb{Z}$

$$
n_{0, a+m \delta}=n^{\prime} .
$$

This implies (2.3) and (2.4) immediately.

Finally we come to show (vi). Suppose $a, b \in \Phi_{0}$ is a prenilpotent pair and $p, q \in F$. Then

$$
\left[\tilde{u}_{a+m \delta}(p), \tilde{u}_{b+n \delta}(q)\right]=\prod_{\substack{i, j \in \mathbb{N}-\{0\} \\ i a+j b \in \Phi_{0}}} \tilde{u}_{i(a+m \delta)+j(b+n \delta)}\left(C_{i j, a+m \delta, b+n \delta} p^{i} q^{j}\right) .
$$

Applying the homomorphism $\alpha$ on both sides, we obtain

$$
\left[\bar{u}_{a}\left(p t^{m}\right), \bar{u}_{b}\left(q t^{n}\right)\right]=\prod_{\substack{i, j \in \mathbb{N}-\{0\} \\ i a+j b \in \Phi_{0}}} \bar{u}_{i a+j b}\left(C_{i j, a+m \delta, b+n \delta} p^{i} q^{j} t^{i m+j n}\right) .
$$

On the other hand, it follows from the commutator formula of Chevalley groups that for arbitrary $x, y \in F\left[t, t^{-1}\right]$,

$$
\left[\bar{u}_{a}(x), \bar{u}_{b}(y)\right]=\prod_{\substack{i, j \in \mathbb{N}-\{0\} \\ i a+j b \in \Phi_{0}}} \bar{u}_{i a+j b}\left(C_{i j a b}^{\prime} x^{i} y^{j}\right),
$$

where the index $i$ and $j$ are taken in the same order as in (2.16). Comparing the above two identities, we obtain that for all $m, n \in \mathbb{Z}$

$$
C_{i j, a+m \delta, b+n \delta}=C_{i j a b}^{\prime},
$$

which gives rise to the identities (2.5) and (2.6) immediately.

Proposition 2.4. There exists a surjective homomorphism $\beta: \tilde{G}(F) \rightarrow G\left(F\left[t, t^{-1}\right]\right)$ whose kernel is contained in the centre of $\tilde{G}(F)$.

Proof. Following Proposition 2.1 and Lemma 2.3, we can define a homomorphism $\beta: \tilde{G}(F) \rightarrow G\left(F\left[t, t^{-1}\right]\right)$ by

$$
\beta\left(\tilde{u}_{a+m \delta}(q)\right)=u_{a}\left(q t^{m}\right), \quad a \in \Phi_{0}, m \in \mathbb{Z}, q \in F .
$$

Since $F\left[t, t^{-1}\right]$ is a Euclidean domain, $G\left(F\left[t, t^{-1}\right]\right)$ is generated by $u_{a}(x)$ for all $x \in F\left[t, t^{-1}\right]$ and $a \in \Phi_{0}$ (see [12], p. 115). Hence $\beta$ is surjective. Moreover, let $A d^{\prime}$ be the adjoint representation of $G\left(F\left[t, t^{-1}\right]\right)$, then one can easily check that the following diagram is commutative.

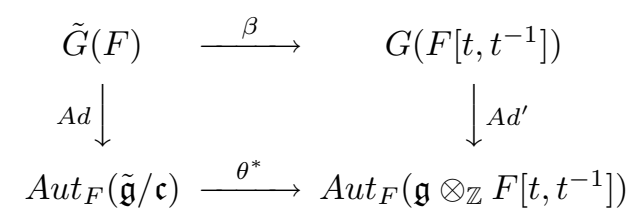

Hence we have

$$
\operatorname{ker} \beta \subseteq \operatorname{ker}\left(\theta^{*} \cdot A d\right)=\operatorname{ker} A d,
$$

which implies that $\operatorname{ker} \beta$ lies in the centre of $\tilde{G}(F)$. 
Remark. Peterson and Kac have mentioned (see [10]) that $\tilde{G}(F)$ should be a central extension of $G\left(F\left[t, t^{-1}\right]\right)$ but no proof is given.

Proposition 2.5. There exists a one-to-one correspondence between the irreducible projective representations over algebraically closed fields of $\tilde{G}(F)$ and those of $G\left(F\left[t, t^{-1}\right]\right)$.

Proof. Suppose $\rho: \tilde{G}(F) \rightarrow P G L(V)$ is an irreducible projective representation of $\tilde{G}(F)$, where $V$ is a finite dimensional vector space over an algebraically closed field. Then it follows from Proposition 2.4 and Shur's lemma that $\rho(\operatorname{ker} \beta)$ is contained in the centre of $P G L(V)$, which is trivial. Hence there exists a unique homomorphism $\rho^{\prime}: G\left(F\left[t, t^{-1}\right]\right) \rightarrow P G L(V)$ such that

$$
\rho=\rho^{\prime} \cdot \beta
$$

Moreover, $\rho^{\prime}$ is irreducible because every $G\left(F\left[t, t^{-1}\right]\right)$-submodule of $V$ is also a $\tilde{G}(F)$-submodule and therefore it must be either trivial or equal to $V$ itself. Thus we have a map between the irreducible projective representations of $\tilde{G}(F)$ and those of $G\left(F\left[t, t^{-1}\right]\right)$ sending a representation $\rho$ to $\rho^{\prime}$. On the other hand, given an irreducible representation $\gamma: G\left(F\left[t, t^{-1}\right]\right) \rightarrow P G L(W)$, where $W$ is a finite dimensional vector space over an algebraically closed field, we claim that the composite $\sigma=\gamma \beta$ is also irreducible. In fact, if $W_{1}$ is a subspace of $W$ and is invariant under the action of $\tilde{G}(F)$ via $\sigma$, then it follows from the above argument that there exists a unique representation $\sigma^{\prime}: G\left(F\left[t, t^{-1}\right]\right) \rightarrow P G L\left(W_{1}\right)$ such that for all $g \in \tilde{G}(F)$ and $v \in W_{1}$

$$
\sigma(g)(v)=\sigma^{\prime} \beta(g)(v) .
$$

Since $\beta$ is surjective, we obtain

$$
\gamma\left(g^{\prime}\right)(v)=\rho^{\prime}\left(g^{\prime}\right)(v), \quad g^{\prime} \in G\left(F\left[t, t^{-1}\right]\right), v \in W_{1},
$$

which means that $W_{1}$ is also invariant under the action of $\gamma\left(G\left(F\left[t, t^{-1}\right]\right)\right)$. Hence $W_{1}$ is either trivial or equal to $V$. This implies that $\sigma$ is irreducible. Thus the correspondence between the irreducible projective representations of $\tilde{G}(F)$ and those of $G\left(F\left[t, t^{-1}\right]\right)$ is one-to-one as required.

\section{Minimal Representation Degrees}

In this section, the notation is the same as that in the previous sections. Recall that the rank of an almost simple algebraic group $H$, which is denoted by rank $H$, is the number of its fundamental roots.

Lemma 3.1. Let $H$ be an almost simple algebraic group. Then

$$
\operatorname{dim} H \geq(\operatorname{rank} H+2) \cdot \operatorname{rank} H \text {. }
$$

Proof. This can be routinely checked for each individual type of almost simple algebraic groups (cf. [4]).

Lemma 3.2. Let $H$ be an almost simple algebraic group over an algebraically closed field $K$. If there exists a homomorphism from $G(F)$ to $H$ with Zariski dense image, then

(i) $\operatorname{char} K=0$;

(ii) the root systems of $G$ and $H$ are isomorphic to each other;

(iii) $\operatorname{dim} H=\operatorname{dim} G$. 
Proof. Let $H_{a d}$ be the group of the adjoint type of $H$ and let $\epsilon: H \rightarrow H_{a d}$ be the canonical isogeny. If $\zeta: G(F) \rightarrow H$ is a homomorphism with Zariski dense image, so is the composite $\epsilon \zeta$. Then it follows from the Borel-Tits theorem [1, Theorem A] that there exist a homomorphism of fields $\varphi: F \rightarrow K$ and an isogeny $\xi:{ }^{\varphi} G(K) \rightarrow H_{a d}$, where ${ }^{\varphi} G(K)$ is the group obtained from the base change through $\varphi$, such that

$$
\epsilon \zeta(g)=\xi\left(\varphi^{\circ}(g)\right), \quad g \in G(F),
$$

where $\varphi^{\circ}: G(F) \rightarrow{ }^{\varphi} G(K)$ is the canonical homomorphism induced by $\varphi$ (see [1] for the notations). Hence (i) results from the fact that $\varphi$ is non-trivial. The properties (ii) and (iii) come directly from $[3, \S 2.5]$.

Lemma 3.3. Let $H$ be a normal subgroup of $G\left(F\left[t, t^{-1}\right]\right)$. If $H$ contains $G(F)$, then $H=G\left(F\left[t, t^{-1}\right]\right)$.

Proof. For each $a \in \Phi_{0}$ and $q \in F^{*}$, let

$$
h_{a}(q)=u_{a}(q) u_{-a}\left(-q^{-1}\right) u_{a}(q) u_{-a}(1) u_{a}(-1) u_{-a}(1),
$$

which lies in $H$. Then Lemma 2.3 yields

$$
h_{a}(q) u_{a}(x) h_{a}(q)^{-1}=u_{a}\left(q^{2} x\right), \quad x \in F\left[t, t^{-1}\right] .
$$

Moreover, taking $q \in F^{*}-\{ \pm 1\}$, we have

$$
u_{a}(x)=h_{a}(q) u_{a}\left(\left(q^{2}-1\right)^{-1} x\right) h_{a}(q)^{-1} u_{a}\left(\left(q^{2}-1\right)^{-1} x\right)^{-1},
$$

which lies in $H$ since $H$ is a normal subgroup. Since $G\left(F\left[t, t^{-1}\right]\right)$ is generated by the elements $u_{a}(x)$ for all $a \in \Phi_{0}$ and $x \in F\left[t, t^{-1}\right]$, this implies that $H$ is equal to $G\left(F\left[t, t^{-1}\right]\right)$.

Proposition 3.4. Let $\rho$ be a non-trivial representation of $G\left(F\left[t, t^{-1}\right]\right)$. Then

(i) $\operatorname{deg} \rho \geq \operatorname{rank} G+1$;

(ii) the base field of $\rho$ must be of characteristic zero.

Proof. Taking into account the natural homomorphism from a general linear group to its projective group, we may assume that $\rho: G\left(F\left[t, t^{-1}\right]\right) \rightarrow P G L_{n}(K)$ is a nontrivial projective representation over an infinite field $K$ without loss of generality. Then (i) follows from [3, Proposition 3.9] and Lemma 3.1. Now Lemma 3.3 implies that the restriction of $\rho$ to $G(F)$ is non-trivial, hence there exists a non-trivial homomorphism of fields from $F$ to $K$ by [1, Theorem 10.3]. This gives rise to (ii).

Theorem 3.5. A simply connected Chevalley-Demazure group scheme $G$ is of type $S L_{n}$ for some $n \geq 1$ if and only if there exists a linear or projective representation $\rho$ of $G\left(F\left[t, t^{-1}\right]\right)$ such that $\operatorname{deg} \rho=\operatorname{rank} G+1$.

Proof. If $G$ is of type $S L_{n}$ for some $n \geq 1$, then the natural embedding $G\left(F\left[t, t^{-1}\right]\right)$ $\rightarrow G L_{n}(F(t))$ is a representation of degree $\operatorname{rank} G+1$, where $F(t)$ is the fractional field of $F\left[t, t^{-1}\right]$. Vice versa, suppose $G\left(F\left[t, t^{-1}\right]\right)$ has a representation $\rho$ whose degree is $\operatorname{rank} G+1$. Without loss of generality we may assume that $\rho: G\left(F\left[t, t^{-1}\right]\right) \rightarrow P G L_{n}(K)$ is a nontrivial projective representation over an algebraically closed field $K$. We first show that the Zariski closure $\overline{\rho(G(F))}$ of $\rho(G(F))$ in $P G L_{n}(K)$ is connected. Let $\overline{\rho(G(F))}$ be the connected component of $\overline{\rho(G(F))}$ which contains the identity element of $P G L_{n}(K)$ and let $\eta$ be the natural homomorphism from $\overline{\rho(G(F))}$ to its quotient group $\overline{\rho(G(F))} / \overline{\rho(G(F))}$. Then 
$G(F) / G(F) \cap \operatorname{ker}(\eta \rho)$ is a finite group since $\overline{\rho(G(F))} / \overline{\rho(G(F))}$ is finite. Note that $G(F)$ does not contain any proper normal subgroup of finite index. Hence

$$
\rho(G(F)) \subseteq \operatorname{ker} \eta=\overline{\rho(G(F))^{\circ}},
$$

which implies immediately the connectedness of $\overline{\rho(G(F))}$.

We claim now that the dimension of $G$ is less than or equal to the dimension of $\overline{\rho(G(F))}$. In fact, since $G(F)$ is equal to its commutator subgroup (cf. [12]), so is $\overline{\rho(G(F))}$. Hence, if we denote by $R$ the solvable radical of the algebraic group $\overline{\rho(G(F))}$, then the quotient group $\overline{\rho(G(F))} / R$ is a semisimple algebraic group of positive dimension, which is an almost direct product:

$$
\overline{\rho(G(F))} / R=G_{1}(K) \cdot G_{2}(K) \cdots G_{m}(K),
$$

where $\left\{G_{i}(K) \mid 1 \leq i \leq m\right\}$ is the set of the simple components of $\overline{\rho(G(F))} / R$. Let $G_{i}^{\prime}(K)$ be a simple adjoint algebraic group which is of the same type as $G_{i}(K)$ for $1 \leq i \leq m$. Then there exists an isogeny $\varepsilon$ from $\overline{\rho(G(F))} / R$ to $\prod_{i=1}^{m} G_{i}^{\prime}(K)$. Consider the composition

$$
G(F) \stackrel{\rho}{\rightarrow} \overline{\rho(G(F))} \stackrel{\tau}{\rightarrow} \overline{\rho(G(F))} / R \stackrel{\varepsilon}{\rightarrow} \prod_{i=1}^{m} G_{i}^{\prime}(K) \stackrel{p_{j}}{\longrightarrow} G_{j}^{\prime}(K),
$$

where $\tau$ is the natural homomorphism and $p_{j}$ is the canonical projection of $\prod_{i=1}^{m} G_{i}^{\prime}(K)$ to its $j$-th factor for $1 \leq j \leq m$. Since the homomorphisms $\tau, \varepsilon$ and $p_{j}$ preserve the Zariski density, so is the composite $p_{j} \varepsilon \tau$. In particular, we have for all $1 \leq j \leq m$

$$
\overline{p_{j} \varepsilon \tau \rho(G(F))}=p_{j} \varepsilon \tau(\overline{\rho(G(F))})=G_{j}^{\prime}(K) .
$$

This means that $p_{j} \varepsilon \tau \rho$ is a homomorphism with Zariski dense image. Thus it follows from Lemma 3.2 (iii) that

$$
\operatorname{dim} G=\operatorname{dim} G_{j}^{\prime}(K)=\operatorname{dim} G_{j}(K) \leq \operatorname{dim} \overline{\rho(G(F))} / R \leq \operatorname{dim} \overline{\rho(G(F))}
$$

This implies by Lemma 3.1 that

$$
(\operatorname{rank} G+2) \operatorname{rank} G \leq \operatorname{dim} \overline{\rho(G(F))} \leq \operatorname{dim} P G L_{n}(K)=n^{2}-1 .
$$

Thus we have, since $n=\operatorname{rank} G+1$,

$$
\operatorname{dim} \overline{\rho(G(F))}=\operatorname{dim} P G L_{n}(K) .
$$

Since $\overline{\rho(G(F))}$ is connected, we obtain that

$$
\overline{\rho(G(F))}=P G L_{n}(K) \text {. }
$$

In other words, $\rho$ is a homomorphism with Zariski dense image. Thus it comes from Lemma 3.2 (ii) that $G$ must be of type $S L_{n}$.

Proof of the main theorem. We assume, without loss of generality, that $\rho: \tilde{G}(F) \rightarrow$ $P G L_{n}(V)$ is a non-trivial irreducible projective representation, where $V$ is a vector space over an algebraically closed field. It follows from Proposition 2.5, as well as its proof, that there exists a representation $\rho^{\prime}: G\left(F\left[t, t^{-1}\right]\right) \rightarrow P G L_{n}(V)$ such that

$$
\rho=\rho^{\prime} \cdot \beta,
$$


where $\beta$ is as in Proposition 2.5. Note that $\rho^{\prime}$ is non-trivial. Hence we have from Proposition 3.4 that

$$
n \geq \operatorname{rank} G+1=\operatorname{rank} \tilde{G} .
$$

Thus (i) is proved. (ii) comes from (3.2) and Proposition 3.4 (ii). Finally, (iii) results from Theorem 3.5 and the fact that $\tilde{\mathfrak{g}}$ is of the non-twisted type $A_{n}^{(1)}$ if and only if $\mathfrak{g}_{\mathbb{C}}$ is of type $A_{n}$ (see [7]), or equivalently the Chevalley-Demazure group scheme $G$ is of the type $S L_{n+1}$. This completes our proof.

\section{REFERENCES}

[1] A. Borel, J. Tits, Homomorphismes "abstraits" de groupes algébriques simples, Annals of Math. 97 (1973), 499-571. MR 47:5134

[2] R. Carter, Y. Chen, Automorphisms of affine Kac-Moody groups and related Chevalley groups, J. Algebra 155 (1993), 44-94. MR 94k:17032

[3] Y. Chen, On rational subgroups of reductive algebraic groups over integral domains, Math. Proc. Camb. Phil. Soc. 117 (1995), 203-212. MR 95m:20051

[4] C.Chevalley, Classification des groupes de Lie algébriques, Notes polycopiées, Inst. H. Poincaré, Paris, 1956/58.

[5] M. Demazure, A. Grothendiek, Schémas en groupes III, Lecture Notes in Math. 153, SpringerVerlag, Berlin-Heidelberg-New York, 1970. MR 43:223c

[6] H. Garland, The arithmetic theory of loop groups, Inst. Hautes Études Sci. Publ. Math. 52 (1980), 5-136. MR 83a:20057

[7] V. Kac, Infinite dimensional Lie algebras, Birkhäuser, Boston, 1983. MR 86h:17015

[8] V. Kac, D. Peterson, Regular functions on certain infinite-dimensional groups, Arithmetic and Geometry, Progress in Math. 36, Birkhäuser, Boston, 1983, p. (141-166). MR 86b:17010

[9] J. Morita, Tits' systems in Chevalley groups over Laurent polynomial rings, Tsukuba J. Math. 3 (1979), 41-51. MR 82a:20050

[10] D. Peterson, V. Kac, Infinite flag varieties and conjugacy theorems, Proc. Natl. Acad. Sci. USA 80 (1983), 1778-1782. MR 84g:17017

[11] A. Pressley, G. Segal, Loop groups, Clarendon Press, Oxford, 1988. MR 88i:22049

[12] R. Steinberg, Lectures on Chevalley groups, Yale Univ. Lect. Notes, 1967. MR 57:6215

[13] J. Tits, Groupes associés aux algèbres de Kac-Moody, Séminaire Bourbaki, 700, Paris, 1987. MR 91c:22034

[14] J. Tits, Uniqueness and presentation of Kac-Moody groups over fields, J. Algebra 105 (1987), 542-573. MR 89b: 17020

Dipartimento di Matematica, Università di Torino, Via C. Alberto 10, 10123 Torino, ITALY

E-mail address: yuchen@dm.unito.it 